\title{
Measurement of the effect of chromaticity and intensity on colour representation parameters of a CRT display
}

\author{
Dr. Klára Wenzel \\ Department of Mechatronics, Optics and Mechanical \\ Engineering Informatics \\ Budapest University of Technology and Economics \\ Budapest, Hungary \\ wenzel@mogi.bme.hu
}

\author{
Ágnes Urbin \\ Department of Mechatronics, Optics and Mechanical \\ Engineering Informatics \\ Budapest University of Technology and Economics \\ Budapest, Hungary \\ urbin@mogi.bme.hu
}

\begin{abstract}
The aim of the measurement detailed in this paper was to measure the just-noticable stimuli of the participating subjects. The stimuli were defined by chromaticity and intensity as the main parameters of the mathematical model. The results show correlance between intensity and the just-noticable stimuli, as described in the Weber-Frechner Law but a contradiction was shown after the analysis in the function of chromaticity. This contradiction can be explained by the difference between the sensitivity of the three cones of the eye.
\end{abstract}

Keywords-Weber-Frechner law; just-noticable stimuli; chromaticity; intensity; CRT display

\section{INTRODUCTION}

The measurement detailed in this paper is the part of a research aiming to observe the influence of coloured lenses on colour vision using tests on displays. Although during this measurement no glasses were used. Since the results of tests created for displays significantly depend on the parameters of the display, in order to be able to focus on the influence of the glasses first we need to know how and which parameters of the display do react for the change of the factors set in the measurements.

\section{CONDITIONS}

During the design process of the experiment the main aim was to avoid the influence of the factors non-defined by us as much as possible.

The main factors of the measurements were intensity and chromaticity in order to correspond with the colour representation parameters of the display. The actual object of the measurements was to find the just-noticable difference between two colour stimuli.

\section{A. Location}

Each measurement was held in the Visual System Laboratory at the Department of Mechatronics, Optics and Mechanical Engineering Informatics at the Budapest University of Technology and Economics. The environmental lighting was shaded and homogeneous illuminance of 400 lux was provided.

\section{B. Exercise}

The measurement started with a neutral grey screen, subjects had to change the colour of a dot in the middle of the screen until they could notice the difference between the background and the dot. The subjects had to sit to a position that provided that the dot was seen in $5^{\circ}$ angle of view.

\section{Subjects}

In the experiment 6 subjects participated. Each of them was good colour observer without any colour vision deficiency. The subjects were in normal mental state, relaxed and they could easily focus on the excercise. The duration of the measurement was set to avoid that the subjects become tired: it took 10 minutes.

\section{Chromaticity}

The chromaticity of the stimuli were the plain R, G and B primers of the display in order to minimize the influence of the noise caused by mixing colours and also in order to be able to calculate with the influence later by mixing any other colours from the primers.

\section{E. Intensity}

In order to set the factor levels of intensity the characteristics of the display was needed. Fig. 1. Fig. 2. and Fig. 3. each show both the measured intensity of the primers and a quadratic polynomial approximation depending on DAC values. 


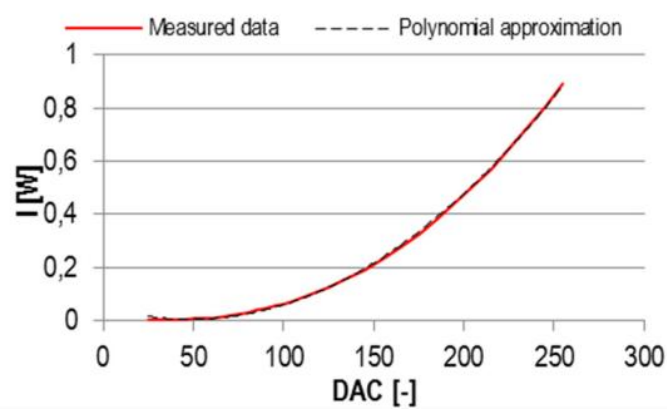

Fig. 1. Measured and approximated intensity of the R primer of the display

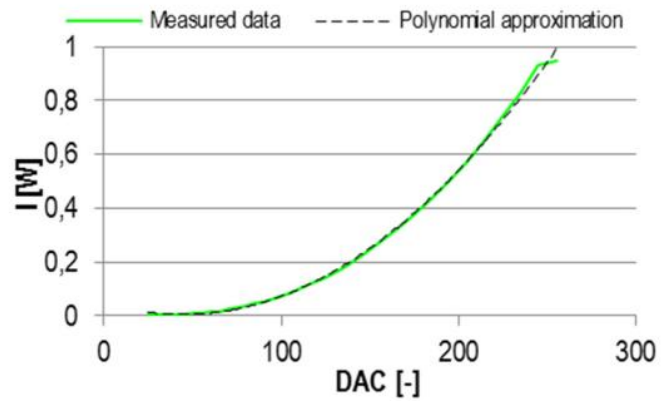

Fig. 2. Measured and approximated intensity of the $\mathrm{G}$ primer of the display

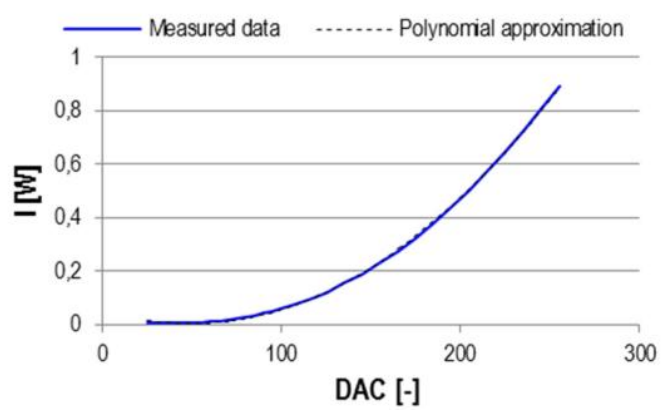

Fig. 3. Measured and approximated intensity of the B primer of the display

The equations of the approximation polynomials are the following:

$$
\begin{aligned}
& I_{G}(D A C)=2 \cdot 10^{-5} D A C^{2}-0,002 \cdot D A C+0,049 \\
& I_{R}(D A C)=2 \cdot 10^{-5} D A C^{2}-0,002 \cdot D A C+0,054 \\
& I_{B}(D A C)=2 \cdot 10^{-5} D A C^{2}-0,002 \cdot D A C+0,057
\end{aligned}
$$

As it can be seen, only the constant coefficients of the functions are different which means that the characteristics of primers of the display are quite similar.
The levels of intensity were selected from the region where the slope of the function is big enough to avoid the influence of high noise.

\section{THE MATHEMATICAL MODEL}

In order to be able to describe the experiment and the results in an exact, compact mode, a mathematical model was set. Since this measurement is a part of a research the mathematical model was created considering further parameters and factor levels so it is basically more complicated than as it was needed for this actual measurement

First define two vectors that contain the values of the 2 main parameters of the measurement.

Let $\underline{B D G}$ be the set of $(R, G, B)$ values that define the colour of the background and $\underline{D O T}$ that contains the $(R, G, B)$ values of the colour of the just-noticable dot.

$$
\begin{aligned}
& \underline{B D G}_{i}(R, G, B), \text { where } i=1 . . n \\
& \underline{D O T}_{j}(R, G, B), \text { where } j=1 . . m
\end{aligned}
$$

The experience is set up of $n$ types of backgrounds and $m$ types of dots. For further calculations use the following unit matrix and unit vectors:

$$
\begin{gathered}
\underline{\underline{e}}_{R G B}=\left[\begin{array}{lll}
1 & 0 & 0 \\
0 & 1 & 0 \\
0 & 0 & 1
\end{array}\right] \\
\underline{e}_{R}=\left[\begin{array}{l}
1 \\
0 \\
0
\end{array}\right]
\end{gathered}
$$

$$
\underline{e}_{G}=\left[\begin{array}{l}
0 \\
1 \\
0
\end{array}\right]
$$

$$
\begin{aligned}
& \alpha+\beta=\chi \\
& \alpha+\beta=\chi .
\end{aligned}
$$

With these the just-noticable stimuli can be defined in the following form: $+\beta=\chi$.

(1)

(1)

$$
\underline{\underline{S T I M}}, j=\left(\underline{D O T}_{j}-\underline{B G D_{i}}\right) \cdot \underline{\underline{e}}_{R G B}
$$

where $\underline{\underline{S T I M}}$ is a matrix with $m \cdot n$ elements of $(R, G, B)$ values. 
The actual values of the measurement can be seen in the following formulas:

$$
\underline{B G D}=\left(\begin{array}{l}
(125,125,125) \\
(185,185,185) \\
(245,245,245)
\end{array}\right)
$$

The dots were set in order to generate stimuli that contain only one primer of the display so always one component was increased from the background. Table 1. shows the average result of the measurements.

TABLE I. THE JUST-NOTICABLE COLOUR STIMULI DEPENDING ON THE BACKGROUND AND THE COLOUR OF THE DOT

\begin{tabular}{|c|c|c|c|}
\hline & $(\mathbf{1 2 5}, \mathbf{1 2 5}, \mathbf{1 2 5})$ & $\mathbf{( 1 8 5 , 1 8 5 , 1 8 5 )}$ & $\mathbf{( 2 4 5 , 2 4 5 , 2 4 5 )}$ \\
\hline $\mathbf{R}$ & $(3,0,0)$ & $(4,0,0)$ & $(4,0,0)$ \\
\hline $\mathbf{G}$ & $(0,2,0)$ & $(0,2,0)$ & $(0,3,0)$ \\
\hline $\mathbf{B}$ & $(0,0,4)$ & $(0,0,5)$ & $(0,0,6)$ \\
\hline
\end{tabular}

\section{ANALYSIS}

The Weber-Fechner law [1] states the relationship between the magnitude of a stimulus and the intensity of the reference can be described in the form:

$$
\frac{d I}{I}=k
$$

where $d I$ is the difference between the two stimuli, $I$ is the reference and $\mathrm{k}$ is a constant. In order to calculate the actual $k$ constants the characteristics and the approximated functions $I_{R}, I_{G}$ and $I_{B}$ detailed above were used.

$$
\begin{aligned}
& I_{i}=I_{R}\left(\underline{B G D_{i}} \cdot e_{R}\right)+ \\
& +I_{G}\left(\underline{B G D_{i}} \cdot \underline{e}_{G}\right)+I_{B}\left(\underline{B G D_{i}} \cdot \underline{e}_{B}\right) \\
& d I_{i, j}=I_{R}\left(\underline{D O T}_{j_{i}} \cdot e_{R}\right)+ \\
& +I_{G}\left(\underline{D O T}_{j} \cdot \underline{e}_{G}\right)+I_{B}\left(\underline{D O T}_{j} \cdot \underline{e}_{B}\right)-I_{i} \\
& \underline{\underline{k}}_{i, j}=\frac{d I_{i, j}}{I_{i}} \\
& \underline{\underline{k}}=\left(\begin{array}{lll}
0,0294 & 0,0215 & 0,0126 \\
0,0145 & 0,0099 & 0,0091 \\
0,0355 & 0,0249 & 0,0219
\end{array}\right)
\end{aligned}
$$

The results of the measurements were analysed depending on both factors of the experiment.

\section{A. The effect of intensity}

$\mathrm{k} \cdot \mathrm{j}$ values (see Table 2.) show exactly what we expected from the Weber-Fechner law: the standard deviations are one magnitude smaller than the averages and the higher background-intensity requires bigger stimuli.

TABLE II. THE AVERAGE VALUES AND STANDARD DEVIATION OF CONSTANT $K$

\begin{tabular}{|l|l|l|}
\hline & average & standard deviation \\
\hline R & 0,0212 & 0,0069 \\
\hline G & 0,0112 & 0,0024 \\
\hline B & 0,0274 & 0,0058 \\
\hline
\end{tabular}

\section{B. The effect of colour}

Table 2. gives another point of view as it shows the $k$ constants depending on the colour of the stimuli. According to this we could say that the Blue primer gives the most intensive sign since the highest $k$ value belongs to that.

In order to understand this effect define $B R_{L}, B R_{M}$ and $B R_{S}$ as the brightness of the $R, G$ and $B$ primers perceived by the human eye.

$$
B R_{R}=\int_{350}^{750} V(\lambda) R(\lambda) d \lambda
$$

$$
B R_{G}=\int_{350}^{750} V(\lambda) G(\lambda) d \lambda
$$

$$
B R_{B}=\int_{350}^{750} V(\lambda) B(\lambda) d \lambda
$$

where $R(\lambda), G(\lambda)$ and $B(\lambda)$ are the spectral intensity functions of the primers of the display and $V(\lambda)$ is the CIE luminosity function.

Table 3. shows the Brightness values in percentage rate.

TABLE III. RATE OF PERCEPTED BRIGHTNESS OF THE PRIME CHANNELS OF THE DISPLAY

\begin{tabular}{|c|c|c|}
\hline$B R_{R}$ & $B R_{G}$ & $B R_{B}$ \\
\hline $44,08 \%$ & $100 \%$ & $13,77 \%$ \\
\hline
\end{tabular}

The values show that the Green channel sends the brightest perceived sign. According to the Weber-Fechner law the highest just-noticable stimuli should belong to that however the results of the measurement show the opposite. 
The conclusion of the Weber-Fechner law above assumes that the sensitivity of the receptors are the same during the measurements and don't depend on the colour of the stimuli.

Fig. 4. shows the spectral sensitivity of the cones and the spectral intensity of the primers of the display.

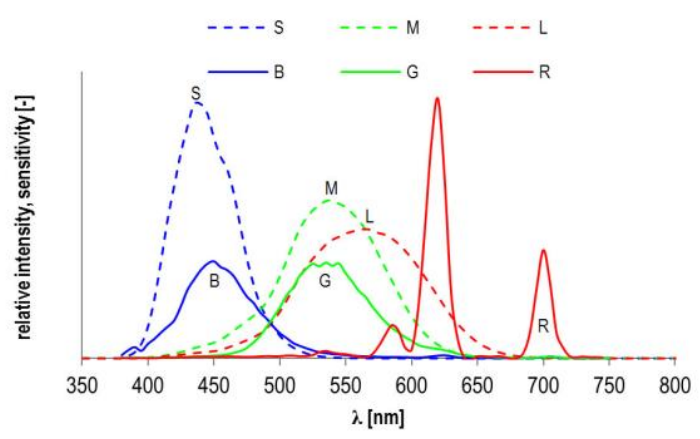

Fig. 4. Spectral sensitivity of the $L, M$ and $S$ receptors and spectral intensity of $R, G$ and $B$ primers of the display

It can be seen that each primer has a spectral intensity corresponding to the sensitivity of a cone so basically each cone can see one of the primer channels

Different stimuli are mainly perceived by different cones (neglecting the overlaps) so the k constants are not comparable without knowing the ratio of the sensitivity of the cones.

Conversely, this context does not only explain the contradiction between the Weber-Fechner law and our results but it also gives information of the sensitivity of the cones.
Since the smallest just-noticable stimuli belongs to the channel to which also belongs the highest perceived brightness, assuming that the stimuli generated by the $G$ primer are perceived by the cone deuteros, it can be said that the deuteros has the best resolution among tritos and protos.

\section{CONCLUSION}

Since the data of the measurements was analysed considering both main factors two conclusions can be stated.

The main result we shall establish is that the contradiction between the Weber-Fechner law and the data of the measurements was caused by the difference between the sensitivity of the cones depending on colours. Additional result is that the sensitivity depending on intensity of the perceived light did correspond with the Weber-Fechner law.

\section{References}

[1] Selig Hecht, The visual discrimination of intensity and the WeberFechner law, 1924

[2] Dr. Ákos Antal, Dr. Krisztián Samu, A monitoros színlátásvizsgálat hazai elterjedése, OPTIKAI MAGAZIN 16:(2) pp. 54-55., 2012

[3] Dr. Klára Wenzel, Ábrahám Gy., Kucsera I., Kovács G., Soterius M.: Possibilities of colour adaptation, CIE Symposium '99, Budapest, 1999.

[4] Dr. Klára Wenzel: Optics of Colors, Womenomics, GE Women's Network Lighting Hungary, Budapest, 2010.07.02.

[5] Dr. Klára Wenzel: Individual Characteristics of Colour Vision, Colour Dynamics ' 88 , Budapest, November 2-6, 1988 